\title{
Rapport de gestion 2012: dynamisme en équilibre
}

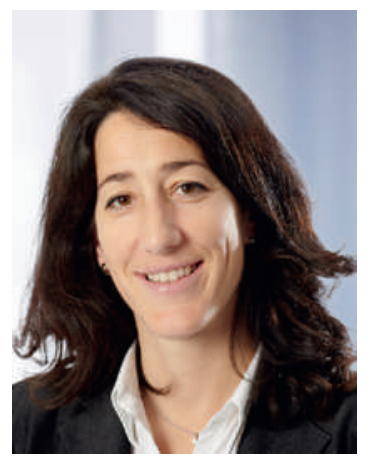

Le temps est venu de faire un retour en arrière sur l'année écoulée. Le rapport annuel est dès à présent disponible sur le site www.fmh.ch ou sous forme papier et offre une vue complète de ce qu'a accompli la FMH au cours de cette période sous le titre harmonieux de «Dynamisme en équilibre».

Chimiquement parlant, qualifier une activité annuelle d'équilibre dynamique laisse comprendre que l'exercice a donné lieu simultanément à des réactions qui se sont pro- changements à l'échelle microscopique! Au travers de ce rapport annuel, nous vous présentons ainsi les principaux faits et illustrons les approches stratégiques qui ont permis d'atteindre cet équilibre ainsi que les chiffres auxquels cela a donné lieu pour 2012.

Vous pourrez ainsi revisiter notamment le dossier des soins intégrés et du Managed care, suivre l'évolution de l'introduction de la nouvelle structure tarifaire SwissDRG et de la réforme du financement hospitalier, vous rappeler l'engagement de la FMH dans le cadre des négociations tarifaires pour la médecine ambulatoire, apprécier les diverses initiatives prises en matière de protection des patients et du secret médical, réaliser l'implication active de la FMH en matière d'informatique médicale et de cybersanté, vous remémorer la création

\section{La FMH peut être comparée à une structure en mouvement qui poursuit sa mission tout en recherchant de manière constante à assurer l'équilibre.}

duites dans des directions opposées mais qui ont ainsi permis d'atteindre finement l'équilibre nécessaire au bon fonctionnement de la structure. A la lecture du rapport annuel, vous comprendrez que la FMH peut être comparée à une structure en mouvement qui poursuit sa mission tout en recherchant de manière constante à assurer l'équilibre des forces qui la composent.

Dans un contexte de la santé en pleine évolution où, à la fois les enjeux politiques, mais également les attentes des ac- d'une nouvelle organisation dédiée à la qualité (ASQM), repenser aux mesures de prévention que la FMH n'adresse pas qu'aux patients mais aussi au corps médical, revivre l'élection des membres du comité et du président de la FMH, penser au soutien de la FMH à l'initiative populaire «Oui à la médecine de famille» et observer l'évolution de la statistique en terme de démographie médicale.

Enfin, pour avoir une vision complète de l'activité de l'Institut suisse pour la formation médicale postgraduée et

\section{Si on ne constate pas de modification fondamentale à l'échelle macroscopique, on peut faire état d'une foison d'activités et de changements à l'échelle microscopique.}

teurs ainsi que celles des patientes et des patients vont croissant, les pressions diverses sont à même de déstabiliser plus ou moins fortement cet équilibre. L'art de maintenir l'équilibre dynamique consiste donc à entreprendre les actions nécessaires pour faire un juste contrepoids et offrir stabilité et maîtrise au système.

Continuons donc notre analyse chimique de la situation; si on ne constate ainsi pas de modification fondamentale de la structure et de la manière de faire à l'échelle macroscopique, on peut réellement faire état d'une foison d'activités et de continue (ISFM), je vous encourage à compléter votre lecture du rapport de gestion que l'ISFM a publié et que vous retrouverez sous www.siwf.ch

Je vous en souhaite une excellente lecture.

Anne-Geneviève Bütikofer, lic. iur., secrétaire générale de la FMH 